\section{Association of "cup-like" blasts morphology with NPM1 mutation in acute myeloid leukemia}

DOI: $10.1515 / \mathrm{rrlm}-2015-0021$

\section{To the Editor:}

Acute myeloid leukemia (AML) is a clonal disease of hematopoietic progenitor cells, characterized by increased proliferation, inhibition of apoptosis, maturation arrest and a low number of mature cells in peripheral blood. Many chromosomal and molecular abnormalities described in AML have diagnostic and prognostic role, some of them being associated with treatment resistance and unfavorable evolution (1). Without minimizing the importance of genetic testing in acute myeloid leukemia, we want to emphasize that, in this case, the morphology of blasts was essential in guiding the diagnostic investigations.

We report a case of AML in a young patient without chromosomal abnormalities, but with a particular morphological appearance of leukemic blast cells ("cup-like" nuclei) which guided the molecular testing and thus facilitated the diagnosis of molecular anomalies important for patient's prognosis. Blast cell morphology should become a criterion for selecting the patients with AML that should be investigated for targeted mutation analysis.

A female patient, aged 46, presented to the gynecologist for the check-up of an intrauterine device. Routine investigations revealed leukocytosis. She was admitted in the hematology department for diagnosis and treatment. Blood cell count was performed on a Sysmex XT-1800i automatic analyzer. Leukemic cells were characterized morphologically in peripheral blood and bone marrow smears using May Grunwald
Giemsa (MGG) and cytochemical stains (Periodic Acid Schiff - PAS, peroxidases and esterases). Immunophenotyping of peripheral blood blast cells was performed on a Becton Dickinson FACSCanto II flow cytometer using BD FACS Diva software v 6.1.3., CE-IVD reagents compatible with the system. Discrimination of cell populations was performed by the method Forward Scatter / Side Scatter and CD45 expression / Side Scatter. The panel used included the following monoclonal antibodies: CD3, CD7, CD11b, CD14, CD15, CD19, CD33, CD34, CD36, CD45, CD56, CD64, CD79a, CD117, MPO, HLA-DR.

Cytogenetic analysis was performed on GTG-banded chromosomes obtained from unstimulated bone marrow cultures ( $24 \mathrm{~h}$ and $48 \mathrm{~h})$. For microscopic analysis an Axio Imager Z1 Zeiss motorized microscope (Carl Zeiss, Germany) equipped with a CCD camera was used. Ikaros software (Metasystems, Germany) was used for karyotyping. Fluorescence in situ hybridization (FISH) studies were performed using a PML/RARA (promyelocytic leukemia/retinoic acid receptor alpha) Translocation Probe (Cytocell) applied on a cytogenetic slide obtained from $48 \mathrm{~h}$ bone marrow culture. Isis software (Metasystems) was used for image analysis. Molecular analysis used real-time polymerase chain reaction (RT-PCR) was performed on ABI 9700 platform for NPM1 and FLT3 genes.

Informed consent for research and publication of data was obtained from the patient according to the Declaration of Helsinki, revised in 2000 to Edinburgh.

The 46 year old female patient, without significant medical history or toxic environmental exposure, with hyperleukocytosis and blast cells in peripheral blood found on routine check-up was admitted on suspicion of acute leukemia. The 
asymptomatic patient presented performance status 0 , moderate gingival hypertrophy without organomegaly. The complete blood count showed marked leukocytosis (WBC $=112,740 / \mathrm{mmc})$, 95\% blast cells, mild thrombocytopenia (PLT = 97,000/mmc), normal hemoglobin level $(\mathrm{Hgb}=$ $12.8 \mathrm{~g} / \mathrm{dl}$ ). The peripheral blood smear showed medium sized, monomorphous blast cells with myeloid, poorly differentiated aspect, non-granular or finely granular cytoplasm, irregular nucleus, "cup-like" or giant nucleolus. The bone marrow smear revealed $85 \%$ infiltration with blast cells, polymorphic, small and medium sized, non-granular or finely granular cytoplasm and giant "cup-like" nuclei (figure 1). All blast cells were negative for Periodic Acid Shiff reaction, while peroxidase reaction was strongly positive.

Flow cytometric analysis (peripheral blood $95,800 \mathrm{cell} / \mathrm{mm}^{3}$ ) - identified $91 \%$ myeloid precursors with microgranular character and phenotype: CD45 + low, + int CD117, CD34 +/- (50\%), CD33 +, HLA-DR -, CD38int, MPO + low. All the other markers evaluated were negative. The same sample contained $6.5 \%$ lymphocytes with no atypical features, $1.8 \%$ granulocytes, and

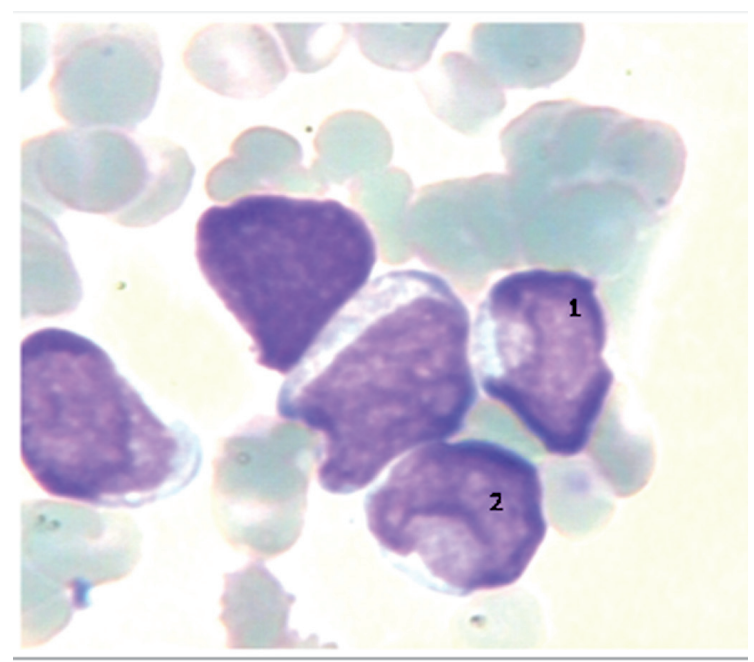

Figure 1 - Bone marrow aspirate - polymorphic blast cells, with giant "cup-like" nuclei (MGG x 1000)
$0.05 \%$ monocytes. The phenotypic profile was suggestive for acute promyelocytic leukemia, microgranular variant (the malignant promyelocytes are characterized by the lower / loss of CD34 and HLA-DR expression) but not diagnostic for APL (HLA-DR negative AML without specific PML / RAR $\alpha$ mutation are described). The morphology of the blasts was not highly suggestive for APL, but there are some reports in literature of APL with "cup-like" appearance. The difference between MPO appearance/intensity may be explained by the fact that flow cytometric analyses were performed from peripheral blood and cytochemistry from bone marrow.

Bone marrow cytogenetics: No clonal chromosome abnormalities were detected on GTG banding. FISH testing (PML/RARA Translocation Probe) showed no chromosome rearrangements involving the RAR $\alpha$ locus (normal fluorescent pattern signals).

Molecular investigations have identified a tetranucleotidic insertion on $\mathrm{C}$-terminal region of the gene NPM1 in the absence of FLT3 gene mutation (absence of ITD mutation).

The diagnosis of AML1 FAB with NMP1 mutation was based on molecular tests suggested by the particular morphology of the blast cells nuclei - "cup-like".

NPM1 gene mutation is the most common genetic abnormality described in acute myeloid leukemia (2). Kroschinsky et al found an incidence of $84.9 \%$ of cup like blast cells in either FLT3 or NPM1 mutation or both in patients with AML compared to $58.1 \%$ in AML not presenting this characteristic morphology (3).

Chen et al. found a $64 \%$ incidence of mutations in "cup-like" AML versus $20 \%$ in the control group AML1 without nuclear invagination. (4) The difference lies in the percentage of "cup-like" blasts used as a criterion for studies conducted by the two authors $(\geq 5 \%$ in Kroschinsky's study and $\geq 10 \%$ a second study). 
Even if the results of studies have sometimes been contradictory (e.g. number of "cup-like" nuclei, morphological FAB type of AML, response to treatment, etc.) most of them showed that "cup-like" nuclear invagination is associated with the loss of HLA-DR expression and the absence of chromosomal abnormalities in classical cytogenetics. Nuclear invagination has been described in microgranular acute promyelocytic leukemia, myelo-monocytic leukemia, acute monocytic AML and nonmonocytic AML.

Some studies showed no impact on response rate or survival parameters of the "cup-like" morphology as an independent prognostic factor (3), but single NPM1 mutation (FLT3-ITD free association) in cup-like AML is a good prognostic factor in AML with normal karyotype (5) being proposed by Vardiman for WHO classification of myeloid neoplasms and acute leukemia (6).

"Cup-like" AML patients presenting NPM1 gene mutation are generally females, with high leukocyte count and increased percentage of bone marrow blast cells with low CD34 expression, HLA-DR negative and normal karyotype. The absence of FLT3-ITD mutation gives good therapeutic results (2, 7-9).

Molecular studies for diagnosis and prognosis are desirable in all patients with AML; if these are not possible, the selection of patients for molecular investigations should be done according to a testing algorithm which can include morphological criteria (for example blast cell morphology with nuclear invagination ("cup like").

Take-away message: Without minimizing the importance of genetic testing in acute myeloid leukemia, we want to emphasize the importance of classic explorations, such as the easy accessible cytomorphology investigation of blasts, sometimes with clinical impact.

\section{Nicoleta Berbec ${ }^{1 *}$, Anca Roxana Lupu ${ }^{1,2}$, Silvana Angelescu ${ }^{1,2}$, Diana Mandescu ${ }^{1}$, Maria Bari ${ }^{2}$}

\section{Coltea Clinical Hospital \\ 2. Carol Davila University of Medicine and Pharmacy}

*Corresponding author: Nicoleta Berbec, e-mail:
nicole_olt@yahoo.com

Conflicts of interest

The authors declare that they have no conflict of interest.

Received: $5^{\text {th }}$ November 2014; Accepted: $28^{\text {th }}$ May 2015; Published: $7^{\text {th }}$ June 2015

\section{References}

1. Berbec N, Papuc S, Tutulan-Cunita A, Angelescu S, Lupu A, Arghir A. Molecular characterization of complex chromosomal change in de novo acute myeloid leukemia; a case report. Rev Romana Med Lab. 2013;21(1):59-65. DOI: 10.2478/rrlm-2013-0018

2. Falini B, Mecucci C, Tiacci E, Alcalav M, Rosati R, Pasqualucci L et al.; GIMEMA Acute Leukemia Working Party. Cytoplasmic nucleophosmin in acute myelogenous leukemia with a normal karyotype [published correction appears in N Engl J Med. 2005;352:740]. N Engl J Med. 2005 Jan;352(3):254-66. DOI: 10.1056/ NEJMoa041974

3. Kroschinsky FP, Schäkel U, Fischer R, Mohr B, Oelschlaegel U, Repp R et al., for the DSIL (Deutsche Studieninitiative Leukämien) Study Group. Cuplike acute myeloid leukemia: new disease or artificial phenomenon? Haematologica. 2008 Feb;93(2):283-6. DOI: 10.3324/haematol.11669

4. Chen W, Rassidakis GZ, Li j, Routbort M, Jones D, Kantarjian $\mathrm{H}$ et al. High frequency of NPM1 gene mutations in acute myeloid leukemia with proeminent nuclear invaginations(,,cuplike nuclei”). Blood. 2006 Sep;108(5):1783-4. DOI: 10.1182/ blood-2006-03-014340

5. Park BG, Chi HS, Jung S, Park CJ, Kim DY, Lee JH et al. Association of cup-like nuclei in blasts with FLT3 and NPM1 mutation in acute myeloid leukemia. Ann Hematol. 2013 Apr;92(4):451-7. DOI: 10.1007/ s00277-012-1645-5

6. Vardiman J, Thiele J, Arber D, Brunning R, Borowitz M, PorwitA et al. The 2008 revision of the World Health Organization (WHO) classification of myeloid 
neoplasms and acute leukemia: rationale and importantchanges. Blood. 2009;114(5):937-51. DOI: 10.1182/ blood-2009-03-209262

7. Schnittger S, Schoch C, Kern W, Mecucci C, Tschulik C, Martelli MF, et al. Nucleophosmin gene mutations are predictors of favorable prognosis in acute myelogenous leukemia with a normal karyotype. Blood. 2005 Dec;106(12):3733-9. DOI: 10.1182/ blood-2005-06-2248

8. Verhaak RG, Goudswaard CS, van Putten W, Bijl MA, Sanders MA, Hugens W et al. Mutations in nu- cleophosmin (NPM1) in acute myeloid leukemia (AML): association with other gene abnormalities and previously established gene expression signatures and their favorable prognostic significance. Blood. 2005 Dec;106(12):3747-54. DOI: 10.1182/ blood-2005-05-2168

9 Thiede C, Koch S, Creutzig E, Steudel C, Illmer T, Schaich $\mathrm{M}$ et al. Prevalence and prognostic impact of NPM1 mutations in 1485 adult patients with acute myeloidleukemia(AML). Blood. 2006 May;107(10):401120. DOI: 10.1182/blood-2005-08-3167 